\title{
Extracellular Serotonin Levels Change with Behavioral State but Not with Pyrogen-induced Hyperthermia
}

\author{
Lynn O. Wilkinson, ${ }^{1, a}$ Sidney B. Auerbach, ${ }^{2}$ and Barry L. Jacobs ${ }^{1}$ \\ ${ }^{1}$ Program in Neuroscience, Department of Psychology, Princeton University, Princeton, New Jersey 08544 and \\ 2Department of Biological Sciences, Rutgers University, New Brunswick, New Jersey 08855
}

\begin{abstract}
Extracellular 5-HT in the anterior hypothalamus/preoptic area (AH/POA) and caudate nucleus of the freely moving cat was measured using in vivo brain microdialysis. Administration of 8-OH-DPAT, a 5-HT ${ }_{1 \mathrm{~A}}$ receptor agonist that decreases 5-HT neuronal activity, decreased extracellular $5-\mathrm{HT}$ in both brain areas. Extracellular 5-HT levels were also examined in relationship to the sleep-wake cycle, because previous data from our laboratory have indicated that behavioral state is the primary determinant of 5-HT neuronal discharge. As with 5-HT neuronal discharge, extracellular 5-HT was increased during active behavioral states and decreased during somnolent periods. These first two sets of findings confirm the ability of the microdialysis technique to measure physiological fluctuations in extracellular 5-HT levels and support the hypothesis that neuronal discharge is a major determinant of extracellular 5-HT levels. Levels of the 5-HT metabolite 5-hydroxyindole acetic acid (5-HIAA) in the AH/POA were also responsive to changes in behavioral state and administration of 8-OH-DPAT, though fluctuations in extracellular 5-HIAA were less robust and temporally delayed. Finally, extracellular 5-HT and 5-HIAA were examined in the AH/POA during fever induced by systemic injection of the synthetic pyrogen muramyl dipeptide. Previous data from our laboratory have indicated that $5-\mathrm{HT}$ neuronal activity is unaffected by this manipulation, though 5-HT has been implicated specifically in thermoregulation. Pyrogen-induced hypothermia produced no specific change in 5-HT efflux, because any changes noted could be accounted for by behavioral state changes. These data are consistent with the hypothesis that the brain serotonergic system is closely linked to the sleep-wake-arousal cycle. However, extracellular 5-HT may be involved in thermoregulatory processes as part of a global role in modulating neuronal activity in coordination with the behavioral state of the animal.
\end{abstract}

Received Sept. 7, 1990; revised Apr. 10, 1991; accepted Apr. 11, 1991.

This work was supported by National Institute of Mental Health Grant MH 23433 and Air Force Office of Scientific Research Grant 87-0301 to B.L.J., by National Science Foundation Grant BNS-87814 and U.S. Public Health Service Grant RR 007085-23 to S.B.A., and by a National Science Foundation predoctoral fellowship to L.O.W. We thank Dr. Keith Martin for assistance with the dialysis technique.

Correspondence should be addressed to Sidney B. Auerbach, Department of Biological Sciences, Rutgers University, P.O. Box 1059, New Brunswick, NJ 088551059.

a Present address: Merck, Sharp and Dohme, Neuroscience Research Centre, Terlings Park, Eastwick Road, Harlow, Essex, CM20 2QR, UK.

Copyright (C) 1991 Society for Neuroscience $0270-6474 / 91 / 112732-10 \$ 03.00 / 0$
In the mammalian CNS, 5-HT has been implicated in a variety of behaviors and physiological processes, including cardiovascular regulation, sleep, pain modulation, and stress. However, single-unit studies have shown that the discharge of 5-HT neurons is positively correlated with the level of behavioral arousal and is unaffected by manipulations of these environmental/ physiological variables. No single environmental manipulation or physiological change has yet been discovered during which the slow and regular discharge of these neurons is perturbed independently of changes in behavioral state (for review, see Fornal and Jacobs, 1988). This suggests that 5-HT is not directly involved in the specific processes cited above.

This conclusion is based upon the assumption that release of 5 -HT is determined primarily by changes in neuronal discharge. However, there could be exceptions to this. For example, 5-HT synthesis and breakdown may be physiologically modulated (Fernstrom and Wurtman, 1971; Luine and Rhodes, 1983), and this could be an important independent factor in regulating 5-HT release (Auerbach and Lipton, 1985). In addition, 5-HT release may be controlled by activation of receptors located presynaptically on nerve endings of 5-HT neurons (for review, see Chesselet, 1984). From this perspective, 5-HT neurons may subserve a variety of different functions, with the regular discharge of 5-HT neurons providing a steady stimulus for initiation of exocytosis, which is then subject to local modulation.

As suggested by both drug and lesion experiments, thermoregulation is one physiological function that may be regulated by 5-HT (reviewed in Myers, 1980). For example, the fever induced by administration of bacterial pyrogens can be attenuated by prevention of 5-HT synthesis (Harvey and Milton, 1974), lesions of the raphe nuclei (Kadlecova et al., 1977), administration of the 5-HT receptor antagonist cyproheptadine (Kandasamy, 1977), or whole-brain depletion of 5-HT with the specific neurotoxin 5,7-dihydroxytryptamine (Matuszek and Ishikawa, 1981). In addition, depletion of 5-HT stores in the primary thermoregulatory center of the anterior hypothalamus/ preoptic area $(\mathrm{AH} / \mathrm{POA})$ compromises the compensatory thermoregulatory response to a cold environment in the monkey (Waller et al., 1976) or rat (Myers, 1975). However, single-unit studies of 5-HT neurons in the dorsal raphe nucleus, a 5-HT nucleus providing a significant innervation of the AH/POA (Van de Kar et al., 1980), indicate that 5-HT neuronal discharge is unchanged during thermoregulatory challenges (Fornal et al., 1987). In this latter study, cats were administered the synthetic pyrogen muramyl dipeptide while brain temperature and 5-HT neuronal discharge were measured. In response to this manipulation, during which brain or body temperature was often in- 
creased by $2^{\circ} \mathrm{C}$, there was no change in either the basal discharge rate of 5-HT neurons, or the fundamental state-related discharge pattern of these neurons (Fornal et al., 1987). These cells were also unresponsive to environmental heating that induced panting and significantly increased brain/body temperature.

The single-unit studies suggest that fever induction and thermoregulatory mechanisms are not regulated by changes in 5-HT neuronal discharge in the dorsal raphe nucleus. However, the 5-HT input to the AH/POA might play a role in thermoregulation through two mechanisms. First, there could be an increase in the discharge of other 5-HT neuronal groups with inputs to the AH/POA. Indeed, the median raphe nucleus (nucleus centralis superior in cat) is an important source of 5-HT in the anterior hypothalamus (Van de Kar and Lorens, 1979). Second, 5-HT released in the $\mathrm{AH} / \mathrm{POA}$ could be regulated independently of 5-HT neuronal discharge during thermoregulatory responses. A local increase in 5-HT relcase might be sufficient to produce thermoregulatory changes because, in the cat, injection of 5-HT into the AH/POA can cause a temperature increase (Feldberg and Myers, 1965). This effect appears to be mediated through 5-HT receptor activation, because it can be blocked by the 5-HT receptor antagonist methysergide (Komiskey and Rudy, 1977).

The present experiments used the in vivo microdialysis technique (for review, see Marsden, 1984) to examine the regulation of 5-HT release in behaving animals. The first purpose of this study was to investigate the relationship between extracellular 5-HT and behavioral state. The second purpose was to examine extracellular 5-HT in AH/POA thermoregulatory centers during the febrile response to a systemically administered pyrogen, one condition in which terminal modulation of release might play a significant role. In order to test the possibility of a dissociation between 5-HT neuronal discharge and 5-HT release, the expcrimental conditions were identical to those previously used in dorsal raphe nucleus single-unit recordings during pyrogeninduced fever (Fornal et al., 1987).

\section{Materials and Methods}

\section{Dialysis probe}

A concentric-style dialysis probe (Hernandez et al., 1986) was used. The surface for exchange was a $5-\mathrm{mm}$ length of dialysis membrane (cellulose hollow fiber, 0.2-mm o.d., 6000 MW cutoff; Cole Parmer, Chicago, IL), and the probe was constructed using stainless-steel tubing. Relative recovery of $5-\mathrm{HT}$ in vitro at a flow rate of $2 \mu \mathrm{l} / \mathrm{min}$ was $20.5 \pm 1.7 \%$ (mean $\pm \mathrm{SEM} ; n=4$ ).

\section{Implantation of guide cannula}

Cats were anesthetized with pentobarbital $(40 \mathrm{mg} / \mathrm{kg}$, i.p.), and from two to six guide cannulas ( 19 ga) were stereotaxically implanted above the target structures. For experiments in the caudate, the tip of the probe extended to the base of the nucleus. Probe tips were aimed at two sites in caudate: $\mathrm{AP}+15.0, \mathrm{ML} \pm 5.0, \mathrm{DV}-15.0$ from dura, and $\mathrm{AP}+18.5$, $\mathrm{ML} \pm 3.5$, and DV -17.0 from dura. For experiments in anterior hypothalamus, a guide cannula was aimed above the anterior hypothalamus/preoptic area (AH/POA; AP +13.5, ML \pm 2.0$)$. The DV coordinate was adjusted according to the length of the guide cannula so that the tip of a $36-\mathrm{mm}$ probe extending through the guide cannula would reach the final target of $D V+6.0$ from stereotaxic zero. The AH/POA site was chosen to match the region in which 5-HT elicited a reproducible rise in body temperature (Komiskey and Rudy, 1977), and to approximate the region in monkey in which an increase in ${ }^{3} \mathrm{H}-5-\mathrm{HT}$ release has been observed during fever (Myers and Belesin, 1971).

In addition, gross electrodes were implanted to allow a detailed analysis of behavioral state. Two stainless-steel screws for bipolar recording of the electroencephalogram (EEG) were implanted in the skull at $A$ $+27, \mathrm{~L}+10$ and at $\mathrm{A}+17, \mathrm{~L}+10$. Eye movements were monitored through a stainless-steel screw implanted in the frontal bone immedi- ately posterior to the eye, and an indifferent electrode was inserted into the frontal bone above the sinus. Electromyographic activity (EMG) was recorded from the uninsulated tips of two stainless-steel multistranded wires inserted bilaterally into the nuchal muscles. The cat was grounded by a stainless-steel screw implanted into the parietal bone so that the temporalis muscle would cover it. For recording of brain temperature, cats were equipped with glass-bead thermistors (Fenwall; 1.2mm o.d., $50 \mathrm{k} \Omega$ at $25^{\circ} \mathrm{C}$ ) implanted supradurally over parietal cortex. All thermistors were calibrated in a silicone fluid bath to the nearest $0.1^{\circ} \mathrm{C}$ prior to implantation (range, $37.0-41.0^{\circ} \mathrm{C}$ ).

Following surgery, the guide cannulas were plugged with stylets, and cats were returned to their home cages for a minimum recovery period of 1 week. Following this period, cats were acclimated to the test chamber over the course of several days before actual experiments were begun.

\section{Dialysis perfusion}

At the start of an experiment, the dialysis probe was slowly inserted into a guide cannula and cemented firmly in place with dental acrylic. Using a Harvard syringe pump, the probe was perfused with filtered $(0.22 \mu \mathrm{m})$, degassed dialysis solution $(147 \mathrm{~mm} \mathrm{NaCl}, 4 \mathrm{~mm} \mathrm{KCl}, 2.3$ $\mathrm{mM} \mathrm{CaCl}$ ) at a rate of $2 \mu \mathrm{l} / \mathrm{min}$. A counterweighted cable and fluid swivel allowed free movement of the cat within a $65 \times 65 \times 95-\mathrm{cm}$ sound-attenuated booth with Plexiglas door. Dialysis solution was collected at 30-min intervals and analyzed immediately for 5-HT and 5-HIAA content. The levels of 5-HT were very high immediately upon implantation of the dialysis probe, but fell to steady levels within $8 \mathrm{hr}$ following probe implantation. The initially high levels were most probably derived from plasma, because the blood-brain barrier is compromised for approximately $4 \mathrm{hr}$ following probe implantation (Benveniste et al., 1987). Thus, all experiments were initiated no sooner than $8 \mathrm{hr}$ following probe implantation.

\section{Behavioral state ratings}

Electrical potentials from the gross electrodes were led from the animal via a low-noise, shielded cable and slip-ring assembly to a polygraph that produced a permanent paper record of the output. This continuous record of behavioral state was scored according to polygraphic criteria as previously described (see Fornal and Jacobs, 1988). Four behavioral states were defined as follows: active waking (AW) was characterizcd by phasic EMG bursts superimposed on high-amplitude tonic EMG activity, frequent $(>20 / \mathrm{min}$ ) eye movement potentials, with at least two such potentials above $400 \mu \mathrm{V}$, and low-voltage fast (desynchronized) EEG activity; quiet waking (QW) included behavioral periods with no phasic EMG bursts and slightly diminished tonic EMG activity, infrequent or no large eye-movement potentials, and predominantly desynchronized EEG activity; slow-wave sleep (SWS) was characterized by diminished tonic EMG activity, no large eye-movement potentials, and large-amplitude $(200-500 \mu \mathrm{V})$ synchronous EEG with sleep spindles present; rapid eye-movement-sleep (REM) was indicated by desynchronized EEG activity, the absence of tonic EMG activity with occasional phasic twitches present, and intermittent rapid-eye-movement potentials. These behavioral state ratings were given numerical values on a scale of 4 to 1 so that AW was assigned a value of 4 and REM received a value of 1 . Behavioral state was scored by a rater blind to the experimental details. Behavioral-state ratings for each minute of a $30-$ min period were then averaged to obtain a mean score for each sampling period.

\section{Analysis of extracellular 5-HT}

High-pressure liquid chromatography with electrochemical detection was used for analysis of 5-HT levels. Separation of 5-HT from other electroactive compounds was achieved with a $10 \mathrm{~cm} \times 3.2 \mathrm{~mm}$ column with 3- $\mu \mathrm{m}$ ODS packing as the stationary phase (Bioanalytical Systems, Lafayette, IN). The mobile phase composition was $0.15 \mathrm{M}$ chloroacetic acid, $0.12 \mathrm{M} \mathrm{NaOH}, 0.18 \mathrm{~mm}$ EDTA, $20 \mathrm{ml} /$ liter acetonitrile, and $10-$ $15 \mathrm{mg} /$ /iter sodium octane sulfate. Mobile phase was delivered by a Bioanalytical Systems (BAS) model 30A dual-piston pump with two pulse dampeners (Scientific Systems, Inc., State College, PA, and BAS), at a flow rate of $1.0 \mathrm{ml} / \mathrm{min}$.

To enhance the ability to detect coelution of unknown substances with 5-HT, a dual-potentiostat electrochemical detector was used (Princeton Applied Research model 400 EC) with two parallel working electrodes at different potentials. The ratio of the output of the two 
electrodes provided an electrochemical measure of the purity of the 5-HT peak. Choice of applied potentials was adjusted so that one working electrode produced near-maximum oxidation of 5-HT, and the other working electrode produced a chromatographic peak half this size. In most experiments, these two potentials were $590 \mathrm{mV}$ and $520 \mathrm{mV}$, respectively, relative to an $\mathrm{Ag}-\mathrm{AgCl}$ reference electrode. For all samples, the ratio of 5-HT peak heights at maximal and half-maximal potentials was calculated and compared to this ratio in pure 5-HT standards.

Identification and quantification of samples was achieved by comparison to a standard solution containing 5-HT and 5-HIAA and measurement of peak height and elution time. Because metabolite levels were approximately 1000 times greater than the levels of 5-HT, mctabolite oxidation peaks were displayed on a second chart recorder. Basal levels of dialysate 5-HT in the caudate nucleus were frequently below detectable levels. In order to increase the amount of 5-HT recovered in the dialysate, the uptake blocker fluoxetine was added to the dialysis solution $(10 \mu \mathrm{M})$ for all experiments in caudate nucleus. With uptake inhibited, basal levels of extracellular 5 -HT were $2.55 \pm 0.79$ $\mathrm{pg} / 60 \mu \mathrm{l}(n=7)$. Changes in 5-HIAA release were not examined in caudate nucleus, because the addition of uptake inhibitor would be expected to affect the pattern of these results. With local perfusion of fluoxetine, cats cycled normally through sleep/wake stages and appeared undisturbed

\section{Procedures}

Baseline sample collection for all experiments began no sooner than 8 $\mathrm{hr}$ following probe implantation. Drugs were administered following the collection of three to five stable baseline samples. To induce an increase in arousal during sleep/wake experiments (experimenter-induced arousal), the experimenter entered the recording area and playfully aroused the cat for a 30 -min period. During all other periods, cats remained isolated in the experimental chamber, with sample collections occurring every $30 \mathrm{~min}$. In all thermoregulation experiments, because pilot data indicated that the process of probe implantation could itself produce a rise in brain temperature lasting from 6 to $8 \mathrm{hr}$, no experiment was begun until brain temperature was within $0.5^{\circ} \mathrm{C}$ of the measure taken before probe implantation. Following collection of at least five stable baseline samples, muramyl dipeptide was administered $(50 \mu \mathrm{g} /$ $\mathrm{kg}$, i.p.). Temperature measurements were recorded in the middle of each 30-min sample period, and behavioral state was monitored continuously in four of six cats as described above. Brain temperature and extracellular 5-HT release was monitored for at least $6 \mathrm{hr}$ following pyrogen administration. In addition, in order to verify the location of the dialysis probes in the thermoregulatory region of the anterior hypothalamus, some sites were tested with a compound that releases endogenous stores of 5-HT. In this experiment, a bolus of $d, l$-fenfluramine in a concentration of $10 \mu \mathrm{g} / \mu \mathrm{l}$ was perfused through the probe at a rate of $2 \mu \mathrm{l} / \mathrm{min}$ for $30 \mathrm{~min}$ while brain temperature and extracellular 5-HT were monitored.

\section{Data analysis}

Experimenter-induced arousal. The effect of experimenter-induced arousal was determined by comparing extracellular 5-HT and 5-HIAA collected during the manipulation to data from two samples immediately before and one sample following the manipulation. Data were calculated as a percentage of the first two baseline samples. The effect of this manipulation was tested using repeated-measures ANOVA followed by Duncan's multiple range test.

Spontaneous fluctuations in behavioral state. Changes in extracellular 5-HT and 5-HIAA during undisturbed fluctuations in behavioral state were also examined. To reduce the between-animal variability, data for each animal were calculated as a percentage of the mean 5-HT release observed during the experiment. In addition, to insure that data from each cat received equal weighting in the statistical analysis, mean scores for a range of behavioral states were generated for each cat. Paired observations of behavioral state, extracellular 5-HT and 5-HIAA were sorted and averaged by behavioral state groupings so that each experiment generated four blocks of numbers for each cat, representing extracellular 5-HT and 5-HIAA collected when the cat was in behavioral states between 2.0 and $2.49,2.5$ and $2.99,3.0$ and 3.49 , and 3.5 and 4.0. Only one $30-\mathrm{min}$ period with a mean behavioral state rating of less than 2.0 was observed, so these data were excluded from statistical analysis. The mean behavioral state rating within each of these blocks was also determined. The relationship between behavioral state ratings and extracellular 5-HT and 5-HIAA concentrations was tested using Spearman's rank correlation coefficient, and differences in extracellular levels of 5-HIAA and 5-HT in different behavioral state groupings were also examined using repeated-measures ANOVA. In addition, 16 consecutive samples were randomly chosen within each of these experiments, and a time-series regression analysis was performed using the Fuller and Batiste estimate of error structure (SAS Institute, Cary, NC) This analysis preserved the temporal relationships between consecutive samples and calculated the significance of the correlative relationship between extracellular 5-HT and behavioral state over time.

Pyrogen administration. In a separate group of animals, changes in bchavioral state, temperature, and extracellular 5-IIT and 5-HIAA concentrations during pyrogen administration were examined over time using repeated-measures ANOVA followed by Duncan's multiple range test. For this analysis, extracellular 5-HT and 5-HIAA levels were calculated as a percent of the first five baseline samples. In addition, the relationship between behavioral state, temperature, and extracellular 5-HT levels was analyzed by a time-series regression analysis described above, using temperature and behavioral state ratings as independent variables. This analysis was also used to evaluate any correlative relationship between temperature and behavioral state during this manipulation, using temperature as the independent variable.

Fenfluramine administration. Changes in temperature and extracellular 5-HT following fenfluramine administration were calculated as a percentage of the levels measured immediately before fenfluramine administration. Because the time course of this effect was variable, changes in temperature were analyzed in relation to the time of maximal 5-HT release. Differences in extracellular 5-HT levels and temperature following fenfluramine administration were examined using a one-tailed $t$ test, because it was predicted a priori that this compound would increase 5-HT release and cause an increase in brain temperature.

\section{Histology}

Once all probe sites were utilized, the cat was deeply anesthetized with pentobarbital and perfused intracardially with physiological saline followed by $10 \%$ formalin. The brain was then removed, and $50-\mu \mathrm{m}$-thick sections were cut through the cannula track of each probe. The sections were mounted on slides and stained with cresyl violet. Probe localization was determined with the aid of a microscope.

\section{Materials}

All chemicals and solvents were of analytical grade or better. Drugs were obtained from the following sources: $d, l-N$-ethyl- $\alpha$-methyl-3-(trifluoromethyl)benzeneethanamine ( $d, l$-fenfluramine), A.H. Robins Co., Richmond, VA; $( \pm$ )-8-hydroxy-2-(di- $n$-propylamino) tetralin $\mathrm{HBr}(8-$ OH-DPAT), Research Biochemicals Incorporated, Natick, MA; muramyl dipeptide, Sigma, St. Louis, MO; and fluoxetine, Lilly Research Laboratories, Indianapolis, IN. 8-OH-DPAT was dissolved in saline and administered subcutaneously at the back of the neck in a volume of $0.5 \mathrm{ml}$. Muramyl dipeptide was dissolved in saline in a concentration of $50 \mathrm{\mu g} / \mathrm{ml}$ and administered intraperitoneally. Fenfluramine and fluoxetine were dissolved in Ringer's solution and pumped slowly through the dialysis probe $(2 \mu \mathrm{l} / \mathrm{min})$. 8-OH-DPAT and fluoxetine solutions were heated gently with warm water until completely dissolved.

\section{Results}

Regulation of extracellular 5-HT during alterations in 5-HT neuronal discharge

\section{Administration of 8-OH-DPAT}

The discharge rate of 5-HT neurons is dramatically decreased by systemic administration of $5-\mathrm{HT}_{\mathrm{IA}}$ receptor agonists such as 8-OH-DPAT, which act at the somatodendritic autoreceptor (Sprouse and Aghajanian, 1987). Subcutaneous administration of $50 \mu \mathrm{g} / \mathrm{kg}$ of 8-OH-DPAT produced a significant decrease in extracellular 5 -HT in the caudate nucleus $\left(F_{(12,24)}=6.92 ; p<\right.$ $0.0001)$ and in the hypothalamus $\left(F_{(8,32)}=6.28 ; p<0.0001\right)$. As shown in Figure 1, the maximal decrease was approximately $45-50 \%$ in both sites. A higher dose of 8-OH-DPAT $(250 \mu \mathrm{g} /$ $\mathrm{kg}$ ) reduced extracellular 5-HT to a similar level $(50 \%)$ in the caudate nucleus $\left(F_{(12.36)}=12.87 ; p<0.0001\right)$. There was no 


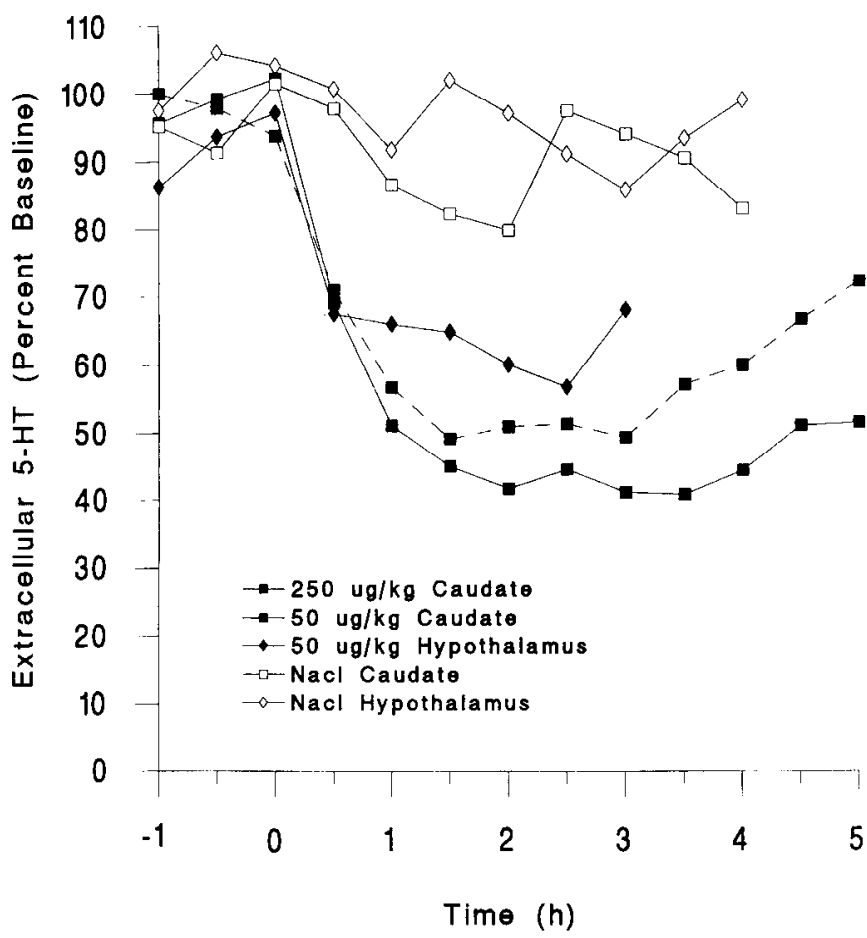

Figure 1. Administration of 8-OH-DPAT produces a decrease in extracellular 5-HT. At time 0, 8-OH-DPAT was administered subcutaneously, and extracellular 5-HT was measured in caudate nucleus [50 $\mu \mathrm{g} / \mathrm{kg}, n=3$ (broken line); $250 \mu \mathrm{g} / \mathrm{kg}, n=4$ (solid line)] and hypothalamus $(50 \mu \mathrm{g} / \mathrm{kg}, n=5)$. Control animals were injected subcutaneously with saline ( $n=3$ in each brain area). For the measurements in the caudate nucleus, fluoxetine was present in the dialysis solution $(10 \mu \mathrm{M})$. SE bars are omitted for clarity.

significant difference in the ability of 50 or $250 \mu \mathrm{g} / \mathrm{kg}$ of $8-\mathrm{OH}-$ DPAT to decrease extracellular 5-HT $\left(F_{(1,7)}=0.566 ; p=0.86\right)$. In all cases, extracellular 5-HT levels were still depressed at 3 $\mathrm{hr}$ following the 8-OH-DPAT injection. Saline administration had no effect on 5-HT concentrations in the caudate nucleus $\left(F_{(10,20)}=0.17 ; p=0.9967\right)$ or the hypothalamus $\left(F_{(10,20)}=0.64\right.$; $p=0.7639$ ).

Extracellular 5-HIAA was also significantly decreased in the AH/POA following 8-OH-DPAT administration $\left(F_{(8,32)}=6.08\right.$; $p<0.0001)$. This decrease was not observed until $1 \mathrm{hr}$ after administration of 8-OH-DPAT (extracellular 5-HIAA was 101.0 $\pm 7.6 \%$ of baseline at $30 \mathrm{~min}$ after $8-\mathrm{OH}-\mathrm{DPAT}$ and $92.2 \% \pm 5.6 \%$ at $1 \mathrm{hr}$ ). Extracellular 5-HIAA levels stabilized at $84.2 \pm 5.2 \%$ of baseline $1.5 \mathrm{hr}$ after 8-OH-DPAT administration and recovered to $88.2 \pm 10.7 \%$ of baseline by $3 \mathrm{hr}$. Saline administration had no effect on 5-HIAA concentrations $\left(F_{(10,20)}=1.59 ; p=0.17\right)$.

\section{Fluctuations in behavioral state}

The discharge rate of 5-HT neurons is correlated with changes in behavioral state (Fornal and Jacobs, 1988). In order to examine the relationship between release and behavioral state, in the first set of experiments, extracellular 5-HT was collected from the caudate nucleus and hypothalamus during experimenter-induced arousal. Thus, once the cat had acclimated to the sampling procedure and was in a stable behavioral state, the experimenter playfully aroused the cat for $30 \mathrm{~min}$. As measured in the caudate nucleus of six cats, this manipulation produced a significant increase in extracellular 5-HT over the level in the

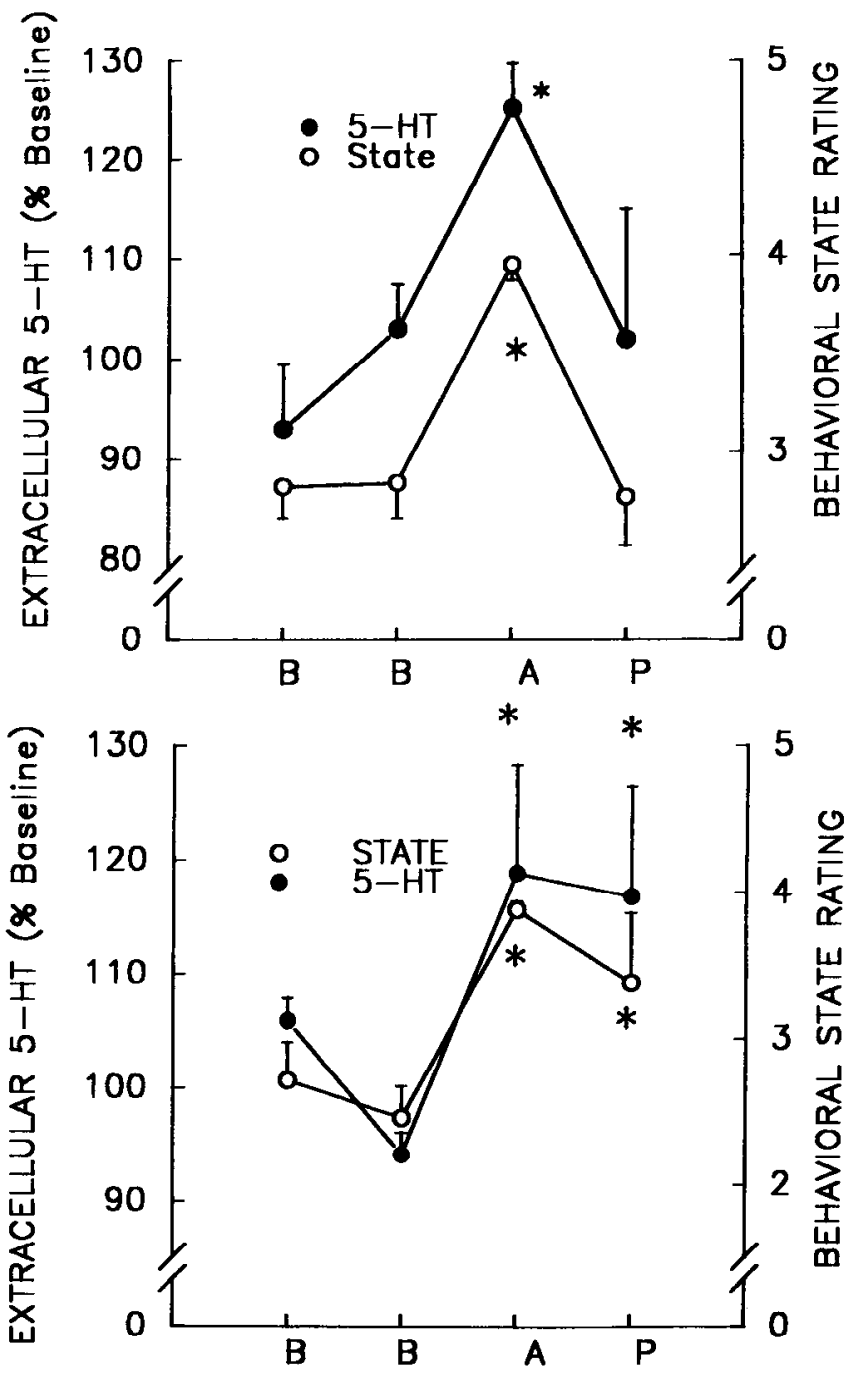

Figure 2. Experimenter-induced arousal produces an increase in extracellular 5-HT. Top, Extracellular 5-HT concentrations in the caudate nucleus and behavioral state ratings before and during a 30 -min period of experimenter-induced arousal. Extracellular 5-HT levels were increased to $127.1 \pm 5.5 \%$ of prearousal basal levels during the arousal period (mean $\pm \mathrm{SE}, n=6$ ). Bottom, Extracellular 5-HT concentrations in hypothalamus during this manipulation. Extracellular 5-HT levels were increased to $118.8 \pm 9.5 \%$ of prearousal basal levels during the arousal period (mean $\pm \mathrm{SE}, n=8$ ). ${ }^{*}$, significantly different from prearousal baseline levels; $p<0.05$, Duncan's multiple range test. $B$, Baseline; $A$, active waking; $P$, post-active waking.

two time points preceding the arousal period $[127.1 \pm 5.5 \%$ during arousal as compared to levels of $95.2 \pm 4.5 \%$ and 104.0 $+3.8 \%$ before arousal $\left(F_{(3,15)}=3.882 ; p<0.03\right)$; see Fig. 2 , top]. Simultaneous verification of the behavioral state changes induced by this manipulation were obtained in four of these cats. Mean behavioral state rating was increased by this manipulation $\left(F_{(3,9)}=26.344 ; p<0.001\right)$ and, during arousal, was $3.9 \pm 0.1$, significantly increased above both pre- $(2.8 \pm 0.2)$ and postarousal $(2.7 \pm 0.2)$ measurements $(p<0.05)$. Extracellular 5-HT was also collected from the anterior hypothalamus of eight cats during experimenter-induced arousal. As shown in Figure 2 (bottom), this manipulation produced a significant increase in extracellular 5-HT above the 5-HT levels in the two preceding samples [118.8 $\pm 9.5 \%$ during arousal as compared to levels of 


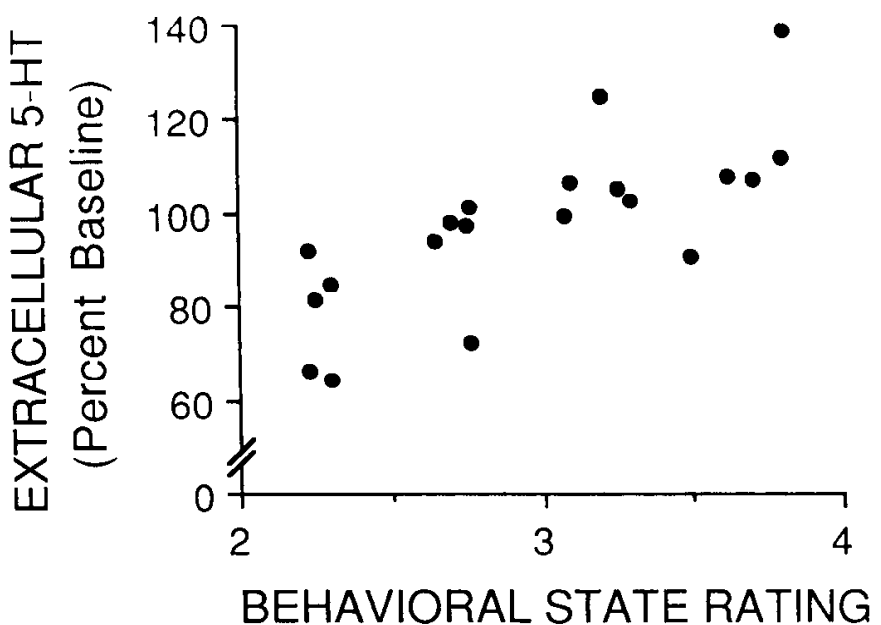

Figure 3. Extracellular 5-HT concentrations in hypothalamus are increased during waking behaviors and decreased during somnolent periods. In five cats allowed to move spontaneously through sleep-wake cycles, the amount of extracellular 5-HT was significantly correlated with behavioral state ratings $\left(r_{s}=0.8169 ; p<0.01\right)$. Thus, extracellular 5-HT levels were higher in periods consisting primarily of waking behavior [with mean behavioral state ratings between $3.0(\mathrm{QW})$ and 4.0 (AW)], and lower in periods that contained periods of slow wave sleep [mean behavioral state ratings between 2.0 (SWS) and 3.0 (QW)]. Mean extracellular 5-HT release during the most active behavioral period (3.54.0 ) was $111.2 \pm 7.8 \%$ of baseline, while mean extracellular $5-\mathrm{HT}$ release during the most somnolent period (2.0--2.5) was $77.8 \pm 5.4 \%$ of baseline.

$105.9 \pm 2.0 \%$ and $94.1 \pm 2.0 \%$ before arousal $\left(F_{(3.2)}=3.584\right.$; $p<0.03)]$. The change in behavioral state produced by this manipulation was measured in five of these cats. Bchavioral state was significantly elevated by this manipulation $\left(F_{(3,12)}=\right.$ $12.131 ; p<0.0006)$, and was significantly above baseline $(2.4$ $\pm 0.2)$ during arousal $(3.8 \pm 0.1)$ and immediately following arousal $(3.4 \pm 0.5)$ in these animals $(p<0.05)$. Extracellular 5 -HIAA levels were not significantly affected by this manipulation in the hypothalamus (data not shown).

In the second set of experiments, changes in hypothalamic 5-HT and 5-HIAA were examined during spontaneous fluctuations in the sleep-wake cycle using data from five cats. As shown in Figure 3, extracellular 5-HT was significantly correlated with behavioral state ratings from each animal $\left(r_{s}=0.8169\right.$; $p<0.01$ ). Mean extracellular 5-HT during the most active behavioral period (ratings of $3.5-4.0$ ) was $42.9 \%$ above mean extracellular 5-HT collected during the most somnolent period, and there was a significant increase in extracellular 5-HT during active behavioral state periods $\left(F_{(3,12)}=5.321 ; p<0.02\right)$. Spccifically, the amount of extracellular 5-HT collected during behavioral states rated between 3.0 and 4.0 was significantly different from that collected during behavioral states rated between 2.0 and 2.99 ( $p<0.05$, Duncan's multiple range test). Timeseries analysis confirmed a significant relationship between behavioral state and extracellular 5-HT $\left(T_{(78)}=2.79 ; p=0.0065\right)$.

Examination of extracellular 5-HIAA revealed that levels of 5-HIAA were significantly correlated with behavioral state, though to a lesser degree than was extracellular 5-HT $\left(r_{s}=\right.$ $0.6383 ; p<0.05$ ). This finding was confirmed by a significant correlation between extracellular 5-HIAA and behavioral state using a time-series analysis $\left(T_{(78)}=2.00 ; p=0.048\right)$, which was again less robust than that seen for 5-HT. The comparative weakness in the strength of the relationship between 5-HIAA and behavioral state can be explained in two ways. First, changes in extracellular 5-HIAA measured in these experiments were dampened and highly autocorrelated. A Durbin-Watson test for autocorrelation revealed that 5-HIAA was significantly autocorrelated $(p<0.05)$ in four of five cats. Thus, extracellular 5-HIAA in any given 30 -min sampling period was not only dependent upon behavioral state, but was also significantly influenced by 5-HIAA levels measured in preceding sampling periods. This is a higher degree of autocorrelation than was observed in measurements of extracellular 5-HT, which was autocorrelated in only one of five cats. Second, a lag was present in the relationship between 5-HIAA and behavioral state. Thus, a time-series regression analysis performed between 5-HIAA and behavioral state in the $30-\mathrm{min}$ period preceding sample collection revealed a stronger relationship between extracellular 5-IIIAA and behavioral state than did time-series regression analysis of simultaneous measurements $\left(T_{(73)}=2.71 ; p<\right.$ 0.01). When extracellular 5-HIAA was related to behavioral states of a longer lag time ( $1 \mathrm{hr})$, this relationship was no longer significant $\left(T_{(68)}=0.72 ; p=0.47, \mathrm{NS}\right)$.

\section{Regulation of extracellular 5-HT during pyrogen-induced fever}

The results summarized in Figure 4 show that there was no significant change in extracellular 5-HT associated with administration of the pyrogen $\left(5-\mathrm{HT}: F_{(16,80)}=1.049 ; p=0.42, \mathrm{NS}\right.$; 5-HIAA: $F_{(16,80)}=0.381 ; p=0.98$, NS; data not shown), despite observations of a significant increase in brain temperature in all six cats $\left(F_{(16,80)}=12.579 ; p<0.001\right)$. Within the first hour, brain temperature $\left(39.5 \pm 0.3^{\circ} \mathrm{C}\right)$ was significantly increased above baseline $\left(38.7 \pm 0.2^{\circ} \mathrm{C} ; p<0.01\right)$. The peak febrile response was reached at $3 \mathrm{hr}$ after pyrogen administration, at which time, brain temperature was $40.1 \pm 0.3^{\circ} \mathrm{C}$, and extracellular $5-\mathrm{HT}$ levels were $107.8+8.6 \%$ of baseline. At no time during the febrile response was extracellular 5-HT significantly above or below the baseline range of $94.5 \pm 4.5 \%$ to $109.3 \pm 6.5 \%$ of prefever levels. The changes in behavioral state ratings observed during this manipulation in the four cats for which these data were available are shown in Figure 4 . In these animals, behavioral state ratings were significantly decreased in the later periods of the febrile phase $\left(F_{(16,48)}=2.364 ; p<0.01\right)$. This effect is due to an increase in slow wave sleep occurring at $6 \mathrm{hr}$ following pyrogen administration. At this time, these cats had a mean behavioral state rating of $2.1 \pm 0.2$, significantly different from all other time points, except those immediately preceding and following the 6-hr measurement. In confirmation of these observations, a time-series regression analysis using temperature and behavior as the independent variables showed no significant relationship between 5-HT and temperature that could not be attributed to a change in behavioral state. Thus, extracellular 5-HT was significantly related to behavioral state ratings $\left(T_{(73)}\right.$ $=3.217, p<0.002$ ), but not significantly related to temperature $\left(T_{(73)}=1.47 ; \mathrm{p}=0.14, \mathrm{NS}\right)$. In addition, there was no significant relationship between behavioral state and brain temperature $\left(T_{\mathrm{(73)}}=1.42 ; p=0.16, \mathrm{NS}\right)$.

Individual examples of the effects of pyrogen administration on behavioral state, temperature, and extracellular 5-HT are shown in Figures 5 and 6. In each example, extracellular 5-HT was responsive to behavioral state changes and was not clearly or consistently related to brain temperature. Thus, in Figure 5 , a rise and fall in extracellular 5-HT that occurred between time 

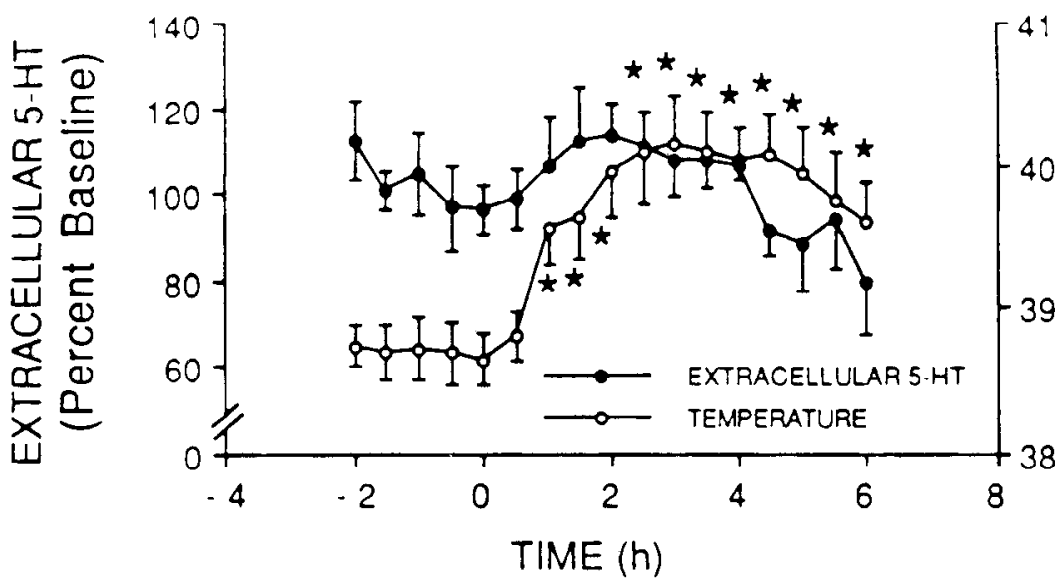
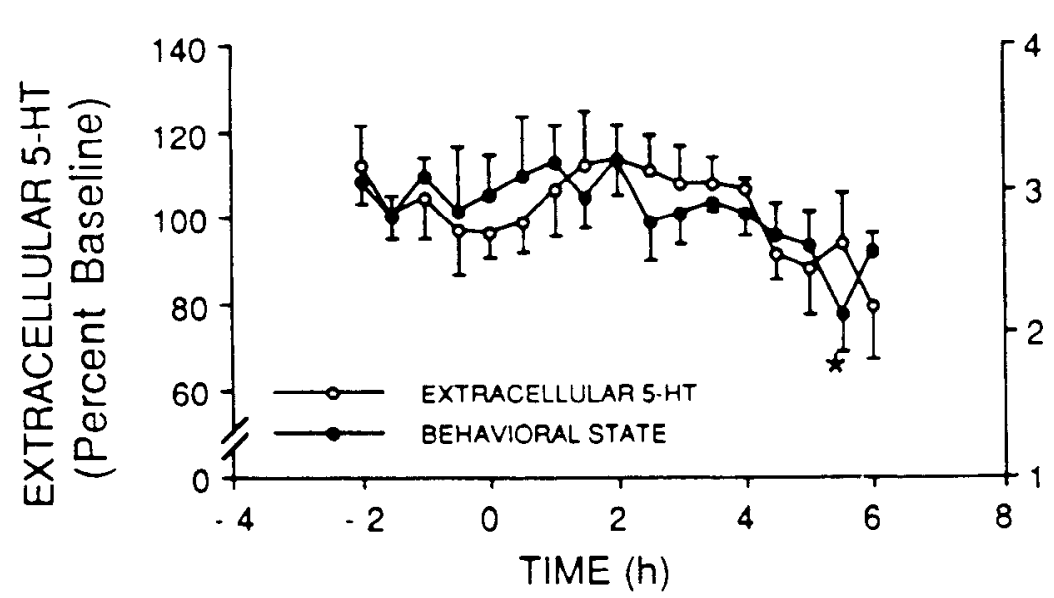

Figure 4. Extracellular 5-HT in the $\mathrm{AH} / \mathrm{POA}$ is not significantly related to fever induced by pyrogen administration. Top, Extracellular 5-HT was not significantly affected by pyrogen administration at time 0 (muramyl dipeptide, $50 \mu \mathrm{g} / \mathrm{kg}$, i.p.) despite the fact that this manipulation induced a significant fever in all cats. Bottom, Changes in extracellular 5-HT are illustrated with behavioral state changes observed during this manipulation. Mean values are shown with SE indicated by the error bars. *, significantly different from baseline; $p<0.05$, Duncan's multiple range test.

periods 2 and 6 can be directly related to a behavioral state change occurring in this period. This animal was activated during the initial phases of fever, displaying shivering and frequent postural adjustments, while in the later phases of fever, the cat became quiescent, and extracellular 5-HT declined. This was the only animal in which a clear change in 5-HT was observed during this manipulation, and it is also the cat that displayed the most robust behavioral response to pyrogen administration. More typical changes in extracellular 5-HT, temperature, and behavioral state are shown in Figure 6. The increases in extracellular 5-HT observed at time points $-0.5 \mathrm{hr}, 0.5 \mathrm{hr}, 4 \mathrm{hr}$, and $5 \mathrm{hr}$ occurred as behavioral arousal spontaneously increased, while there was no clear relationship between extracellular 5-HT and temperature.

In order to verify the location of the probes in a thermosensitive region of the hypothalamus, some sites were tested with the 5-HT-releasing compound $d$, $l$-fenfluramine. Local administration of this drug produced an increase in both extracellular 5-HT levels and brain temperature in all five cats in which this manipulation was examined. At 60 min following the peak change in 5 -HT release, mean brain temperature was $38.9 \pm 0.3^{\circ} \mathrm{C}$, significantly elevated above the basal temperature of $38.5 \pm$ $0.2^{\circ} \mathrm{C}\left(T_{(4)}=2.82 ; p<0.05\right)$. The maximal observed increase in brain temperature was a $0.7^{\circ} \mathrm{C}$ increase, which occurred in the animal with the highest extracellular 5-HT levels following fenfluramine administration $(896.1 \mathrm{pg} / 60 \mu \mathrm{l})$. For the experimental group, peak extracellular 5 -HT was $275 \pm 160 \mathrm{pg} / 60$ $\mu \mathrm{l}$, a greater than 300 -fold increase above baseline levels of 0.76 $\pm 0.3 \mathrm{pg} / 60 \mu \mathrm{l}\left(T_{(4)}=2.76 ; \mathrm{p}<0.05 ;\right.$ Fig. 7$)$. Histological analysis confirmed that the dialysis probes were located in the AH/POA.

\section{Discussion}

The present observations provide several pieces of evidence that 5-HT release in the caudate nucleus and hypothalamus of the cat is highly correlated with changes in the discharge of 5-HT neurons. First, a pharmacological manipulation that suppresses 5-HT neuronal discharge, systemic 8-OH-DPAT administration, produced a large decrease in extracellular 5-HT. Second, extracellular 5-HT was increased during periods of behavioral arousal and decreased during somnolent periods. This is consistent with previous work demonstrating that the discharge of 5-HT neurons is positively correlated with levels of behavioral arousal. Last, in agreement with evidence from single-unit studies, during the febrile state induced by systemic administration of a pyrogen, extracellular 5-HT was not directly correlated with changes in body temperature but, instead, continued to follow changes in level of behavioral arousal. We found no cvidence that extracellular 5-HT levels in the anterior hypothalamus could be dissociated from behavioral state during pyrogen-induced fever.

We first examined extracellular 5-HT release following administration of the somatodendritic autoreceptor agonist $8-\mathrm{OH}-$ DPAT. In the freely moving cat, we found that 5 -HT neuronal 

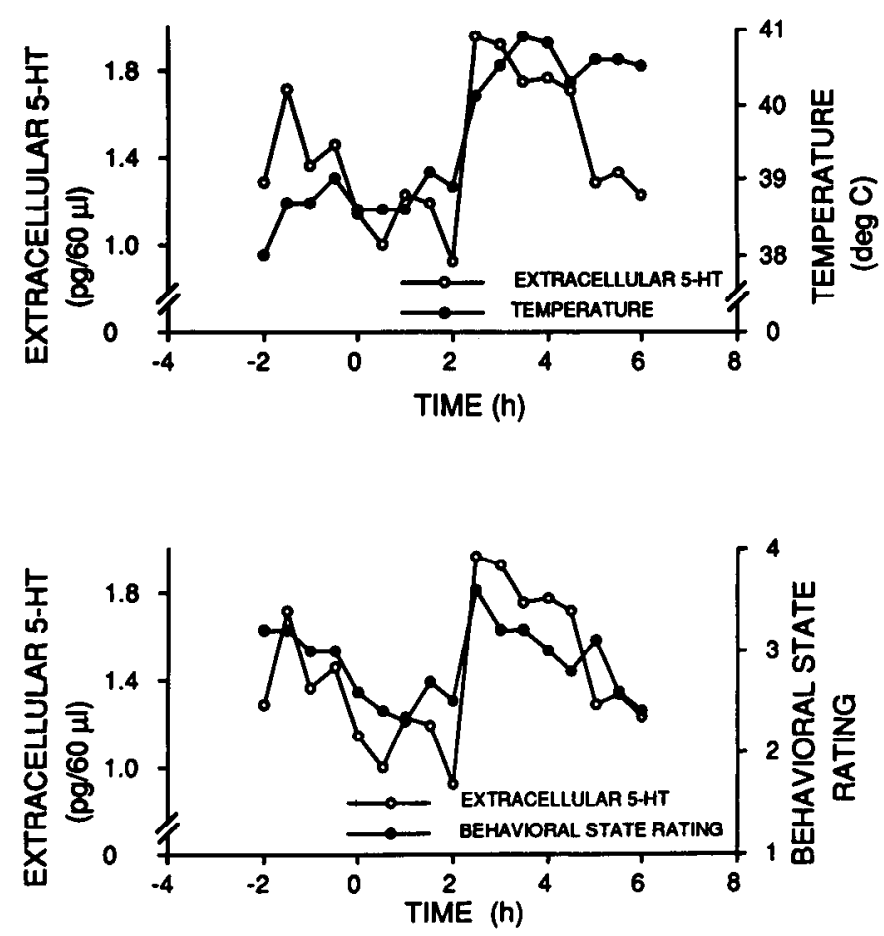

Figure 5. The effects of pyrogen administration on temperature, behavioral state, and extracellular 5-HT in the AH/POA of one cat. Extracellular 5-HT collected in the AH/POA of one cat is illustrated in relationship to changes in brain temperature (top) and behavioral state (bottom) observed after administration of the synthetic pyrogen muramyl dipeptide $(50 \mu \mathrm{g} / \mathrm{kg}$, i.p.) at time 0 . The observed changes in extracellular 5-HT are more closely related to behavioral state changes produced by this manipulation than to brain temperature per se.

activity is completely suppressed for a period of approximately 30 min following this manipulation (C. A. Fornal, L. O. Wilkinson, and B. L. Jacobs, unpublished observations). In the present studies, this treatment resulted in a substantial decrease in extracellular 5-HT in hypothalamus and in the caudate nucleus. This is consistent with the hypothesis that a large fraction of 5-HT in microdialysis samples of extracellular space is neuronal in origin and supports the feasibility of using microdialysis for measuring decreases in 5-HT release during periods of decreased neuronal discharge. In order to determine whether this was a maximal decrease in extracellular 5-HT, a higher dose of 8-OH-DPAT was administered. A subcutaneous dose of 250 $\mu \mathrm{g} / \mathrm{kg} 8-\mathrm{OH}$-DPAT did not produce a significantly greater decrease in extracellular $5-\mathrm{HT}$ than did $50 \mu \mathrm{g} / \mathrm{kg} 8-\mathrm{OH}-\mathrm{DPAT}$, though studics of 5-HT neuronal activity derived using identical conditions indicate that 5-HT neuronal activity is completely suppressed for up to $2 \mathrm{hr}$ following the higher dose of $8-\mathrm{OH}-$ DPAT (Fornal, Wilkinson, and Jacobs, unpublished observations). Thus, the approximately $50 \%$ decrease in extracellular 5-HT levels observed following both doses of 8-OH-DPAT appears to be the maximal observable response to this drug. These findings suggest that approximately $40-50 \%$ of extracellular 5-HT measured by dialysis is unresponsive to complete inhibition of 5-HT neuronal discharge.

This observation is consistent with previous findings that a proportion of extracellular 5-HT is insensitive to manipulations that block 5-HT nerve-terminal depolarization and exocytosis. For example, extracellular 5-HT was decreased approximately
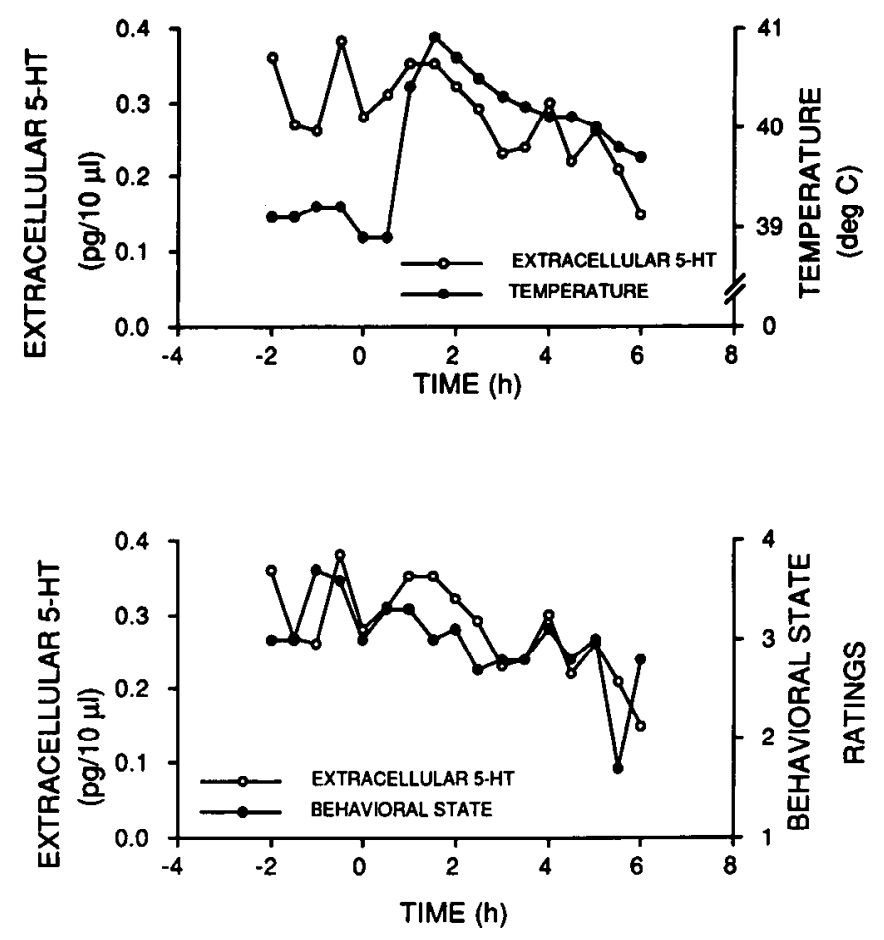

Figure 6. The effects of pyrogen administration on temperature, behavioral state, and extracellular 5-HT from the AH/POA of one cat. Extracellular 5-HT collected in the AH/POA of one cat is illustrated in relationship to changes in brain temperature (top) and behavioral state (bottom) observed after administration of the synthetic pyrogen muramyl dipeptide $(50 \mu \mathrm{g} / \mathrm{kg}$, i.p.) at time 0 . As illustrated in this example, typical changes in extracellular 5-HT observed during this manipulation were slight and correlated with behavioral-state changes, rather than with changes in brain temperature.

$50-70 \%$ in several forebrain regions following local perfusion with tetrodotoxin (Kalen, 1988; Auerbach et al., 1989) and following administration of 5-HT agonist drugs (Kalen, 1988; Auerbach et al., 1989; Sharp et al., 1989). This convergent evidence suggests that a proportion of extracellular 5-HT measured by dialysis does not depend on depolarization of 5-HT nerve terminals. While the possibility that these findings represent an artifact of the dialysis technique cannot be ruled out at present, physiological sources for this residual level of 5-HT in dialysate samples could be non-action potential-dependent release from neurons, platelets, or mast cells (Theoharides et al., 1982).

It is interesting that the proportion of action potential-dependent 5 -HT measured by dialysis is increased to $70-80 \%$ in those experiments that include a 5 -HT reuptake inhibitor in the perfusion fluid (Kalen, 1988; Sharp et al., 1989, 1990). This suggests that addition of a reuptake inhibitor may not only increase the likelihood of a measureable level of 5-HT, but may also increase the degree to which dialysate 5-HT includes 5-HT released from neurons. In our experiments, we observed a greater decrease in extracellular 5-HT in the caudate nucleus (where a 5-HT reuptake inhibitor was included) than we observed in the hypothalamus (where no reuptake inhibitor was used). This again suggests that addition of a reuptake inhibitor may increase the proportion of neuronal 5-HT release in dialysates (though the possibility of an intrinsic difference between caudate and hypothalamus was not addressed in our experiments).

These data form a promising foundation for further exami- 
nation of the physiological control of 5-HT release. Previous work in this laboratory has demonstrated that the discharge of 5-HT neurons is positively correlated with levels of behavioral arousal. The mean firing rate of a 5-HT-containing neuron in the dorsal raphe nucleus during the active waking behavioral state is $3.4 \pm 0.2 \mathrm{spikes} / \mathrm{sec}$. This firing pattern slows to $2.8 \pm$ $0.2 \mathrm{spikes} / \mathrm{sec}$ during quiet waking, and is $1.3 \pm 0.1 \mathrm{spikes} / \mathrm{sec}$ during slow wave slecp (Trulson and Jacobs, 1979). The present experiments demonstrate that this basic relationship between 5-HT neuronal discharge and arousal is paralleled by changes in extracellular 5-HT across the sleep-wake cycle. During periods of arousal, levels of extracellular 5-HT were increased in comparison to somnolent periods in both the caudate nucleus and the hypothalamus. Spontaneous fluctuations in behavioral state occurring during the natural sleep-wake cycle were also paralleled by alterations in 5-HT release. Indeed, in the unanesthetized animal, it appears that a significant proportion of the variability in extracellular 5-HT can be attributed to changes in the behavioral state of the animal. However, changes in extracellular 5-HT appear to be somewhat less dynamic than changes in 5-HT neuronal activity. Thus, while 5-HT neuronal activity during active waking is approximately double that observed during slow wave sleep, in the change from slow wave sleep to active waking, we found a $42 \%$ increase in cxtraccllular 5-HT. Two explanations can be offered for this finding. First, the dialysis technique measures extracellular 5-HT levels and assumes that this is a reflection of changes in synaptic 5-HT. While this assumption has been validated in a number of experiments, changes in this measure might be expected to be less dynamic than those occurring in the synapse. Second, as discussed previously, we found that approximately $50 \%$ of measured 5-HT is not responsive to a complete suppression of 5-HT neuronal activity. The presence of this residual level of 5-HT in dialysate samples would also dampen the measured responses of the system. If the presence of this residual level is corrected for, the relationship of extracellular 5-HT to 5-HT neuronal activity is quite good.

We also observed a significant relationship between extracellular 5-HIAA levels and 5-HT neuronal activity, though this relationship differed from that observed for 5-HT on two grounds. First, state-related changes in extracellular 5-HIAA levels were more dampened and autocorrelated than were the changes observed in extracellular 5-HT. This difference could derive from the different mechanisms by which each of these neurochemicals is cleared from the extracellular space: $5-\mathrm{HT}$ is rapidly cleared from the extracellular space by an efficient uptake process (Iversen, 1974), while 5-HIAA moves across the blood-brain barrier using a far less efficient acid transport process (Wolfson et al., 1974). Second, changes in extracellular 5-HIAA appear to lag behavioral state changes and $5-\mathrm{HT}$ release. A decrease in 5-HT neuronal activity produced by $8-\mathrm{OH}-\mathrm{DPAT}$ did not produce a significant change in extracellular 5-HIAA until $1 \mathrm{hr}$ following drug administration (30 min later than the observed changes in 5-HT). In addition, the strongest relationship between 5-HIAA and behavioral state was revealed when 5-HIAA was examined in relationship to behavioral state changes occurring $30 \mathrm{~min}$ earlier. The consequence of this lag period and a high degree of autocorrelation in the measurement of extracellular 5-HIAA may be that 5-HIAA reflects gradual changes in behavioral state and neuronal discharge across a period of hours rather than those occurring during 30 -min sampling periods.

In summary, the brain microdialysis technique can detect
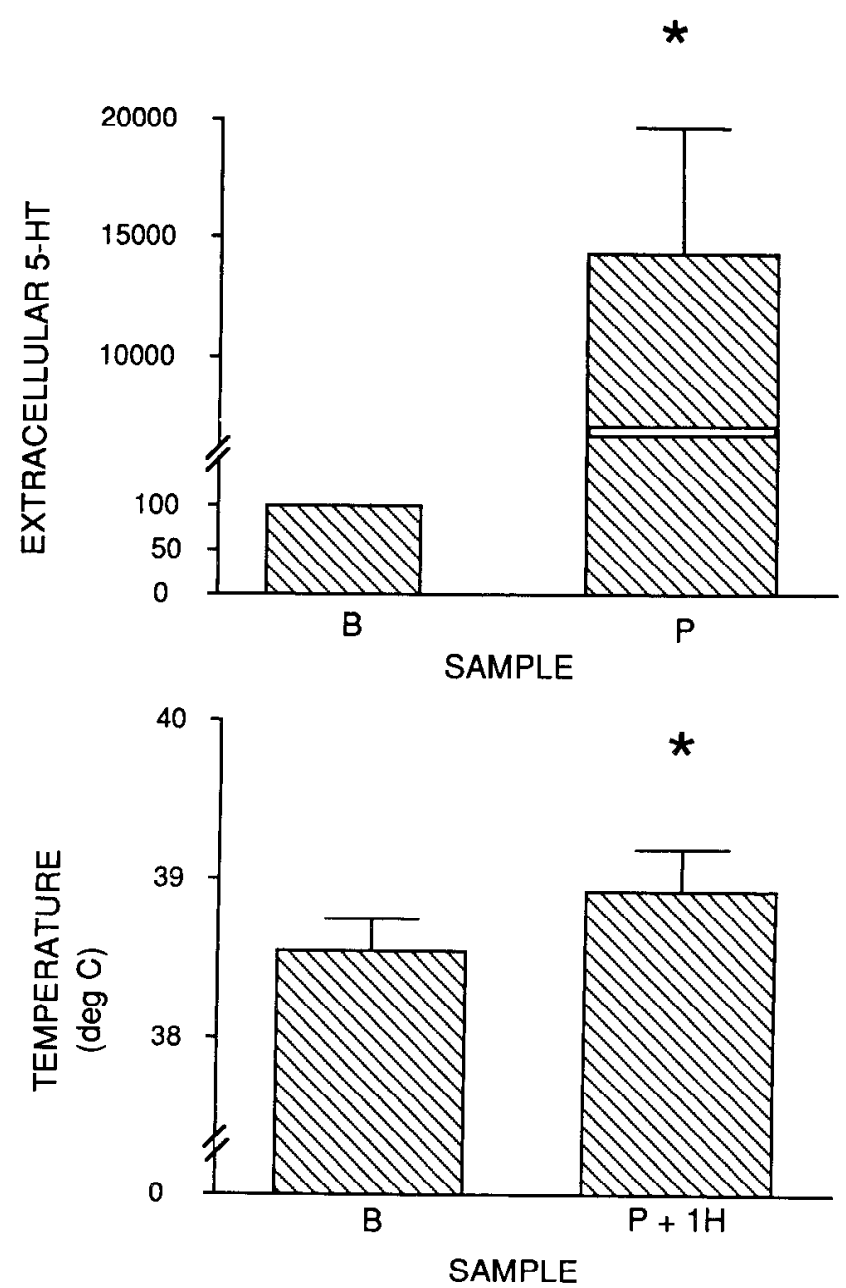

Figure 7. A regional increase in hypothalamic extracellular 5-HT concentrations induced by the addition of $d, l$-fenfluramine to the dialysis Ringer's solution produced a significant increase in brain temperature. Addition of $d, l$-fenfluramine to the dialysis Ringer's solution $(10 \mu \mathrm{g} / \mu \mathrm{l}$ for $20 \mathrm{~min}$ ) produced a large increase in extracellular 5-HT (top) and significantly increased brain temperature (bottom). Brain temperature was elevated from a baseline of $38.5 \pm 0.2^{\circ} \mathrm{C}$ to $38.9 \pm 0.3^{\circ} \mathrm{C}$ (mean $\pm \mathrm{SE}, n=5$ ). *, significantly different from baseline, paired $t$ test. $B$, basal levels; $P$, peak levels of $5-\mathrm{HT} ; P+I H$, temperature $60 \mathrm{~min}$ following the peak 5-IIT release.

behaviorally related changes in extracellular 5-HT in the unanesthetized animal. Within the context of these experiments, extracellular 5-HT fluctuated in parallel with changes in the discharge of 5-HT neurons. The experiments involving fever induction examined a physiological situation in which a dissociation between these two measures might be expected.

We found no consistent relationship between extracellular 5-HT and brain temperature during fever, despite evidence that 5 -HT was sampled in the appropriate brain site. This conclusion is based on the observation that addition of the 5-HT-releasing agent fenfluramine to the dialysis Ringer's solution produced a rcliable increase in brain temperature of a magnitude and time course similar to that previously reported for the local injection of 5-HT (Komiskey and Rudy, 1977). Thus, our data confirm previous findings that large increases in extracellular 5-HT in the $\mathrm{AH} / \mathrm{POA}$ produce temperature elevation, but indicate additionally that endogenous alterations in 5-HT release are not the mechanism through which fever is generated. This obser- 
vation, coupled with the fact that the extracellular concentration of 5-HT necessary to induce a temperature increase in this study and previous examinations (Feldberg and Myers, 1965; Komiskey and Rudy, 1977) was much higher than any observed under physiological conditions, underscores the difficulties inherent in the interpretation and execution of studies based upon regional drug infusion into the brain. Additionally, our data illustrate the importance of an examination of behavioral state changes in experiments designed to determine the role of 5-HT in behavior. Thus, in one animal (see Fig. 5), we did observe an increase in extracellular 5-HT following pyrogen administration. However, because the pattern of the observed increase in 5-HT followed the pattern of behavioral state changes and not temperature, and because this increase was not observed in animals that did not respond behaviorally (see Figs. 4, 6), we could determine that this was a state-related change in extracellular 5-HT. In previous experiments, systematic changes in behavioral state during the thermoregulatory response may have been responsible for reported changes in extracellular 5-HT levels during thermoregulatory challenges (e.g., Myers and Belesin, 1971).

The present data, combined with evidence from single-unit studies in the behaving animal (for review, see Fornal and Jacobs, 1988), are consistent with the hypothesis that the brain 5-HT system is intimately associated with the sleep-wake cycle. In addition, we did not observe a dissociation between extracellular 5-HT levels and 5-HT neuronal discharge during pyrogen-induced fever, suggesting that under these conditions 5-HT release in the anterior hypothalamus is primarily determined by 5 -HT neuronal activity. While the inability to observe a specific change in extracellular 5-HT levels during fever could also be explained by the inherent limitations of the dialysis technique, the clear relationship observed between extracellular 5-HT and behavioral state suggests a difference in the respective strength of the relationship of 5-HT to behavioral state and the mechanisms of fever generation.

Nonetheless, extracellular 5-HT could still play some role in temperature regulation through some mechanism that affects synaptic 5-HT but does not alter extracellular levels. Alternatively, it is possible that extracellular 5-HT may regulatc thermoregulatory processes through a general role in gating neuronal activity in association with the behavioral state of the animal. For example, it has been demonstrated that lesions of the dorsal raphe abolished thermosensitive responses without affecting the baseline firing rate of thermosensitive neurons in the preoptic area (Werner and Bienek, 1985). This effect is similar to the well-described effects of 5-HT on the "signal-to-noise" ratio in somatosensory neurons (Waterhouse et al., 1986), in which iontophoretic application of 5-HT preferentially suppresses evoked neuronal discharge, with minimal perturbation of spontaneous discharge. Alterations in extracellular 5-HT concentrations occurring during the natural sleep-wake cycle may thus affect the sensitivity of thermoregulatory neurons in the hypothalamus. This effect may play some role in the dampening of the thermoregulatory response to hypothalamic warming that occurs during REM sleep (Parmeggiani, 1980). It sccms plausible that 5-HT could play a similar role in other brain areas. A role for 5-HT as a state-sensitive coordinator of brain activity might thus explain the wealth of data implicating the 5-HT system in pain, motor processes, cardiovascular control, and the other diverse behavioral and physiological processes that vary across the sleep-wake-arousal cycle (Fornal and Jacobs, 1988).

\section{References}

Auerbach SB, Lipton P (1985) Regulation of serotonin release from the in vitro rat hippocampus: effects of alterations in levels of depolarization and in rates of serotonin metabolism. J Neurochem 44: $1116-1129$.

Auerbach SB, Minzenberg MJ, Wilkinson LO (1989) Extracellular serotonin and 5-hydroxyindoleacetic acid in hypothalamus of the unanesthetized rat measured by in vivo dialysis coupled to high performance liquid chromatography with electrochemical detection: dialysate serotonin reflects neuronal release. Brain Res 499:281-290.

Benveniste H, Drejer J, Schousboe A, Diemer NH (1987) Regional cerebral glucose phosphorylation and blood flow after insertion of a microdialysis fiber through the dorsal hippocampus in the rat. J Neurochem 49:729-734.

Chesselet MF (1984) Presynaptic regulation of neurotransmitter release in the brain. Neuroscience 12:347-375.

Feldberg W, Myers RD (1965) Changes in temperature produced by micro-injections of amines into the anterior hypothalamus of cats. J Physiol (Lond) 177:239-245.

Fernstrom JD, Wurtman RJ (1971) Brain serotonin content: physiological dependence on plasma tryptophan levels. Science 173:149152.

Fornal CA, Jacobs BL (1988) Physiological and behavioral correlates of serotonergic single-unit activity. In: Neuronal serotonin (Osborne NN, Hamon M, eds), pp 305-345. New York: Wiley.

Fornal CA, Litto WJ, Morilak DA, Jacobs BL (1987) Single unit responses of serotonergic dorsal raphe nucleus neurons to environmental heating and pyrogen administration in freely moving cats. Exp Neurol 98:388-403.

Harvey CA, Milton AS (1974) The effect of parachlorophenylalanine on the response of the conscious cat to intravenous and intraventricular bacterial pyrogen and to intraventricular prostaglandin E1. J Physiol (Lond) 236:14P-15P.

Hernandez L, Stanley BG, Hoebel BG (1986) A small, removable microdialysis probe. Life Sci 39:2629-2637.

Iversen LL (1974) Uptake mechanisms for neurotransmitter amines. Biochem Pharmacol 23:1927-1935.

Kadlecova O, Masek K, Petrovicky P (1977) A possible site of action of bacterial peptidoglycan in the CNS. Neuropharmacology 16:699702.

Kalen P (1988) Regulation of brain stem serotonergic and noradrenergic systems. PhD thesis, University of Lund, Sweden.

Kandasamy SB (1977) Central effect of 5,8,11,14-eicosatetraenoic acid (arachidonic acid) on the temperature in the conscious rabbits. Experientia 33:1626-1627.

Komiskey HL, Rudy TA (1977) Serotonergic influences on brain stem thermoregulatory mechanisms in the cat. Brain Res 134:297-315.

Luine VN, Rhodes JC (1983) Gonadal hormone regulation of MAO and other enzymes in hypothalamic areas. Neuroendocrinology 36 : 235-241.

Marsden CA (1984) Measurement of neurotransmitter release in vivo. Chichester: Wiley.

Matuszek M, Ishikawa Y (1981) Effects of 5,7-dihydroxytryptamine and 6-hydroxy-dopamine on fever response in conscious rats. Pol J Pharmacol Pharm 33:305-312.

Myers RD (1975) Impairment of thermoregulation, food and water intakes in the rat after hypothalamic injections of 5,6-dihydroxytryptamine. Brain Res 94:491-506.

Myers RD (1980) Hypothalamic control of thermoregulation: neurochemical mechanisms. In: Handbook of the hypothalamus. Vol 3 (Morgane PJ, Panksepp J, eds), pp 83-210. New York: Marcel Dekker.

Myers RD, Bclesin DB (1971) Changes in serotonin release in hypothalamus during cooling or warming of the monkey. Am J Physiol 220:1746-1754.

Parmeggiani PL (1980) Temperature regulation during sleep: a study in homeostasis. In: Physiology in sleep (Orem J, Barnes CD, eds), pp 97-143. New York: Academic.

Sharp T, Bramwell SR, Grahame-Smith DG (1989) 5-HT 1 agonists reduce 5-hydroxytryptamine release in rat hippocampus in vivo as determined by brain microdialysis. Br J Pharmacol 96:283-290.

Sharp T, Bramwell SR, Grahame-Smith DG (1990) Release of endogenous 5-hydroxytryptamine in rat ventral hippocampus evoked by electrical stimulation of the dorsal raphe nucleus as detected by dialysis: sensitivity to tetrodotoxin, calcium and calcium antagonists. Neuroscience 39:629-637. 
Sprouse JS, Aghajanian G (1987) Electrophysiological responses of serotonergic dorsal raphe neurons to $5-\mathrm{HT}_{1 \mathrm{~A}}$ and $5-\mathrm{HT}_{1 \mathrm{~B}}$ agonists. Synapse 1:3-9.

Theoharides TC, Bondy PK, Tsakalos ND, Askenase PW (1982) Differential release of serotonin and histamine from mast cells. Nature 297:229-231.

Trulson ME, Jacobs BL (1979) Raphe unit activity in freely moving cats: correlation with level of behavioral arousal. Brain Res 163:135150.

Van de Kar L, Lorens SA (1979) Differential serotonergic innervation of individual hypothalamic nuclei and other forebrain regions by the dorsal and median midbrain raphe nuclei. Brain Res 162:45-54.

Van de Kar LD, Lorens SA, Vodraska A, Allers G, Green M, Van Orden DE, Van Orden LS III (1980) Effect of selective midbrain and diencephalic 5,7-dihydroxytryptamine lesions on serotonin content in in- dividual preopticohypothalamic nuclei and on serum lutenizing hormone level. Neuroendocrinology 31:309-315.

Waller MB, Myers RD, Martin GE (1976) Thermoregulatory deficits in the monkey produced by 5,6-dihydroxytryptamine injected into the hypothalamus. Neuropharmacology 15:61-68.

Waterhouse BD, Moises HC, Woodward DJ (1986) Interaction of serotonin with somatosensory cortical neuronal responses to afferent synaptic inputs and putative neurotransmitters. Brain Res Bull 17: 507-518.

Werner J, Bienek A (1985) The significance of nucleus raphe dorsalis and centralis for thermoafferent signal transmission to the preoptic area of the rat. Exp Brain Res 59:543-547.

Wolfson LE, Katzman R, Escriba A (1974) Clearance of amine metabolites from the cerebrospinal fluid: the brain as a "sink." Neurology 24:772-779. 